\title{
Charged Higgs search at CDF
}

\section{Geumbong Yu*t}

University of Rochester

E-mail: gbyuefnal.gov

I present the recent search for charged Higgs bosons in decays of top quarks in $p \bar{p}$ collisions at $\sqrt{s}=1.96 \mathrm{TeV}$. The charged Higgs is assumed to decay only into $c \bar{s}$, and the charged Higgs mass can be reconstructed using two jets in top quark decays. The search is performed by looking for an anomalous resonance in the dijet invariant mass distribution of the lepton+jets $t \bar{t}$ candidates. In $2.2 \mathrm{fb}^{-1}$ CDF II data, no evidence of charged Higgs boson is observed, hence 95\% C.L. upper limits are placed at $\mathscr{B}\left(t \rightarrow H^{+} b\right)<0.1$ to 0.3 for charged Higgs boson masses of 90 to 150 $\mathrm{GeV} / c^{2}$.

Prospects for Charged Higgs Discovery at Colliders

September 16-19 2008

Uppsala, Sweden

\footnotetext{
* Speaker.

${ }^{\dagger}$ The CDF collaboration
} 


\section{Introduction}

To date there is no evidence of a Higgs boson [1], which is responsible for the masses of fermions and bosons in both standard model (SM) and Supersymmetric theory (SUSY). The Large Hadron Collider is expected to give answers to the unresolved questions in the SM with seven times higher energy than the Tevatron soon. Nevertheless, it is very important not to miss any new physics from existing Tevatron data. Unfortunately the direct production cross-section of such new particle is too small to separate it from the enormous SM processes at Tevatron energy scale. Therefore new particles have been searched in the decays of or in associated production with SM particles. This search was motivated by the possibility that a charged Higgs boson $\left(H^{+}\right)$is involved in top quark decays as predicted in the Minimal Supersymmetric Standard Model (MSSM) [2].

Since the top quark was discovered at the Tevatron Run I program [3], the charged Higgs has been searched in top quark decays. The previous search [4] performed at CDF looked for a deficiency or an excess of the top pair production rates in four different $t \bar{t}$ channels: bblvlv (dilep$\operatorname{ton}^{1}$ ), $b b l v j j$ (lepton+jets with exact $1 b$-tags and $\geq 2 b$-tags) and $b b l v \tau_{h} v$ (lepton+tau). These $t \bar{t}$ channels are categorized by the decay particles of the $W$ bosons. The search assumed five final states of a top quark: $t \rightarrow W b, t \rightarrow H^{+} b$ with $H^{+} \rightarrow \tau^{+} v, c \bar{s}, t^{*} \bar{b}, W h^{0}(\rightarrow b \bar{b})$. The acceptances of each top quark final state resulting in four different $t \bar{t}$ channels were studied using Monte Carlo (MC) simulation samples. In conclusion, the number of observed events was compared to the SM expectation in each $t \bar{t}$ channel, and the differences were translated into the exclusion limit of the MSSM parameter $\left(M_{H^{+}}\right.$vs. $\left.\tan \beta\right)$ as shown in Fig. 1.

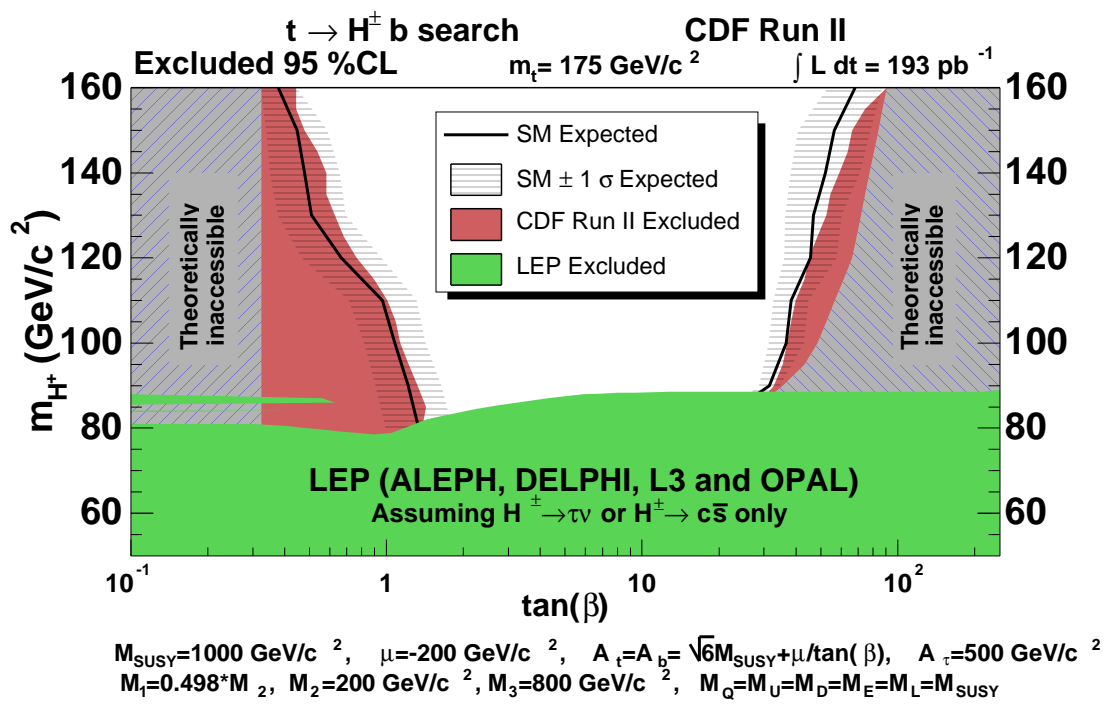

Figure 1: Exclusion limit on the MSSM parameters $\left(m_{H^{+}}, \tan \beta\right)$ plane. This limit is computed with SUSY parameters assuming maximum mass of the $h^{0}$ in reference [5].

Here I present the first direct search for $\mathrm{H}^{+} \rightarrow c \bar{s}$ in top quark decays using fully reconstructed mass $M_{H^{+}}$. The $c \bar{s}$ decay is dominant at small $\tan \beta(\leq 1)$ for low $H^{+}$masses $\left(\leq 130 \mathrm{GeV} / c^{2}\right)$ in

\footnotetext{
${ }^{1}$ Here the lepton implies electron and muon. Tau-lepton is marked separately.
} 
the MSSM. In spite of the tight conditions, this channel provides a good opportunity to reconstruct $H^{+}$. Since the $c \bar{s}$ decay leaves two jets as does the hadronic $W$, the search is performed by looking for a second peak in the dijet mass spectrum in the lepton+jets top quark decays. The lepton+jets channel has the best signal to background ratio, thus it is ideal for charged Higgs search. The treelevel diagram of the lepton+jets $t \bar{t}$ decay involving the charged Higgs boson is shown in Fig. 2. This search uses a data sample with an integrated luminosity of $2.2 \mathrm{fb}^{-1}$ collected by the Collider Detector at Fermilab II (CDF II) [6].

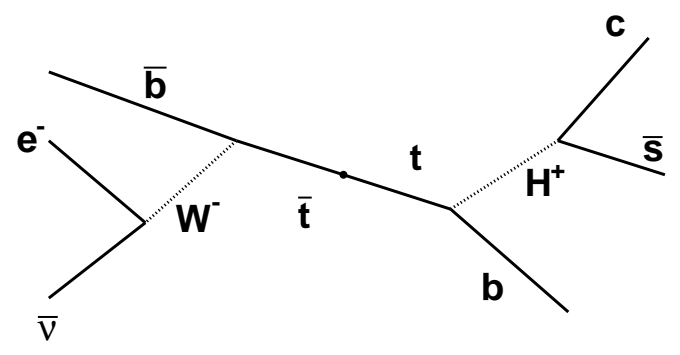

Figure 2: $H^{+} \rightarrow c \bar{s}$ in lepton+jet $t \bar{t}$ events.

\section{Event Selection and Reconstruction}

The $t \bar{t}$ candidates in the lepton+jets channel are selected by requiring an isolated electron with transverse energy $E_{T}>20 \mathrm{GeV}$ or requiring a muon with transverse momentum $p_{T}>20 \mathrm{GeV} / \mathrm{c}$ in the central region, $|\eta|^{2}<1$.0. The missing transverse energy $\left(\mathbb{E}_{T}\right)$ is required to be larger than 20 $\mathrm{GeV}$ for the neutrino. For the four final state quarks in the $t \bar{t}$ decay, at least four jets are required to have $E_{T}>20 \mathrm{GeV}$ in $|\eta|<2.0$. The most energetic four jets are called leading jets, which are used for the $t \bar{t}$ event reconstruction. In addition, at least two of the leading jets are required to have a secondary vertex within them [7] implying a long-lived $b$-hadron from a $b$-quark. The secondary vertex tagged jet is called a $b$-jet, and the other untagged jet is called an h-jet.

Since two $b$-jets are already selected for two $b$-quarks, the $M_{H^{+}}$is reconstructed directly from two h-jets. However, its mass can be improved by reconstructing the $t \bar{t}$ event as a whole. The $t \bar{t}$ event is kinematically reconstructed by assigning the final state objects to the $t \bar{t}$ decay particles. The assignment is evaluated in the form of $\chi^{2}$,

$$
\begin{aligned}
\chi^{2} & =\sum_{i=l, 4 \mathrm{jets}} \frac{\left(p_{T}^{i, \mathrm{fit}}-p_{T}^{i, \mathrm{meas}}\right)^{2}}{\sigma_{i}^{2}}+\sum_{j=x, y} \frac{\left(p_{j}^{U E, \mathrm{fit}}-p_{j}^{U E, \text { meas }}\right)^{2}}{\sigma_{U E}{ }^{2}} \\
& +\frac{\left(M_{l v}-M_{W}\right)^{2}}{\Gamma_{W}{ }^{2}}+\frac{\left(M_{b l v}-M_{t}\right)^{2}}{\Gamma_{t}{ }^{2}}+\frac{\left(M_{b j j}-M_{t}\right)^{2}}{\Gamma_{t}{ }^{2}}
\end{aligned}
$$

In the $\chi^{2}$ function, leptonic decays of the $W$ boson $(l v)$ are constrained to have an invariant mass of $80.4 \mathrm{GeV} / c^{2}$, and both top quark decays ( $b l v$ and $b j j$ ) are constrained to have the same top quark

\footnotetext{
${ }^{2} \eta=-\ln \tan (\theta / 2)$, where $\theta$ is a polar angle with respect to the proton beam direction at CDF
} 
mass of $175 \mathrm{GeV} / c^{2}$. Minimizing the $\chi^{2}$ by MINUIT [8] fit, the measured energies ( $p_{T}^{i \text {,meas }}$ ) of the lepton and four leading jets, and the unclustered transverse energies $\left(p_{j}^{U E \text {,meas }}\right)$ are allowed to vary within their uncertainty. The $U E$ is sum of the extra measured energies, which is not included in the leading jet energies. The $U E$ fit is used to have correct $\mathbb{E}_{T}\left(=-p_{T}^{i, \text { fit }}-p_{j}^{U E \text {,fit }}\right)$. Among the possible assignments, the assignment with the best $\chi^{2}$ is used for this $t \bar{t}$ analysis. Subsequently, the two h-jets reconstruct hadronically decaying charged boson with better resolution.

\section{Backgrounds}

In search for the charged Higgs boson, all the SM processes are considered background. The biggest background is the SM $t \bar{t}$ events, which are about $92 \%$ of the background. The rest of the $\mathrm{SM}$ processes are called non- $t \bar{t}$ backgrounds consisting of diboson $\left(W W^{-}, Z Z, W^{ \pm} Z\right)$, single top, $Z\left(\rightarrow \tau^{+} \tau^{-}\right)+$light flavored jets, multijets and $W+$ jets processes. Among the backgrounds, electroweak processes (SM $t \bar{t}$, diboson, and single top) and $Z+$ jets background have well predicted cross-sections, hence the expected number of electroweak events are estimated directly from the MC simulation study. However, MC program could not reproduce the production rate of jet backgrounds ( $W+$ jets and multijets) due to large theoretical uncertainty. Those backgrounds are estimated directly from the selected data after subtracting the electroweak and $Z+$ jets candidates. Details of the background estimation method can be found in [7]. Assuming $6.7 \mathrm{pb}$ for the SM $t \bar{t}$ production cross-section [9], the numbers of expected and observed events in the $2.2 \mathrm{fb}^{-1}$ data sample are listed in Table. 1.

\begin{tabular}{c|c}
\hline \hline diboson \& single top & $2.6 \pm 0.4$ \\
$W / Z+$ jets \& multijets & $11.4 \pm 7.1$ \\
SM $t \bar{t}$ & $152.6 \pm 25.0$ \\
\hline Observed Events & 200 \\
\hline \hline
\end{tabular}

Table 1: Expected number of events from the SM processes is compared to the observed $t \bar{t}$ candidates in the CDF II data sample of $2.2 \mathrm{fb}^{-1}$. The production cross-section of the SM $t \bar{t}$ process is assumed to be 6.7 $\mathrm{pb}$.

\section{Simulation}

The dijet mass distributions of the $H^{+}, W$, and non- $t \bar{t}$ background are obtained from the MC simulation sample. The $H^{+}$boson in decays of $t \bar{t}\left(\rightarrow b \bar{b} H^{+}(\rightarrow c \bar{s}) W^{-}(\rightarrow l v)\right)$ is generated with $H^{+}$masses of 90 to $150 \mathrm{GeV} / c^{2}$ and zero width of $H^{+}$. The PYTHIA [10] MC program is used to simulate the $H^{+}$sample and the SM $t \bar{t}$, assuming a top quark mass of $175 \mathrm{GeV} / c^{2}$. The diboson and single top processes also use PYTHIA program for the simulation. ALPGEN [11] event generator with the PYTHIA parton showering are used for the simulation of backgrounds involving jets. All the MC samples are realistically simulated to reflect the time dependent operation of the CDF II. 


\section{Improvement on $M_{H^{+}}$}

In the $H^{+}$simulation, the reconstructed $H^{+}$has a significant low mass tail if the $t \bar{t}$ event has extra jets in the final state. The extra jets are mostly hard radiation jets originating from either incoming quarks (initial state radiation) or daughter particles of the $\mathrm{H}^{+}$(final state radiation). The final state radiation jet is close to its mother particle, while the initial state radiation jet is randomly distributed. In order to recover the lost energy due to radiation, the most energetic extra jet (called $5^{\text {th }}$ jet) is merged with the closest leading jet if the $5^{\text {th }}$ jet has $E_{T}>12 \mathrm{GeV}$ in $|\eta|<2.4$, and if $\Delta R\left(=\sqrt{(\Delta \eta)^{2}+(\Delta \phi)^{2}}\right.$, where $\phi$ is an azimuthal angle) between the $5^{\text {th }}$ jet and the nearest leading jet is less than 1.0. This merging process improves the entire $t \bar{t}$ final state energy in the kinematic reconstruction fit and the mass resolution by $5 \%$ in the $120 \mathrm{GeV} / c^{2} H^{+}$events with more than four final jets.

\section{Extracting $\mathscr{B}\left(t \rightarrow H^{+} b\right)$}

In order to extract the components of the dijet mass distribution, a binned likelihood $(L H)$ is constructed employing Poisson probability:

$$
L H=\prod_{i} \frac{v_{i}^{n_{i}} \times e^{-v_{i}}}{n_{i} !} \bigotimes G\left(N_{b k g}, \sigma_{b k g}\right)
$$

Prior probability $\left(P^{i}\right)$ of finding events in the mass bin $i$ comes from a set of simulated dijet mass distributions of $H^{+}, W$, and non- $t \bar{t}$ backgrounds, called templates. The templates are shown in Fig. 3. The Poisson probability in bin $i$ is computed from the prior probabilities, $v_{i}=P_{H^{+}}^{i} \times$ $N_{H^{+}}+P_{W}^{i} \times N_{W}+P_{b k g}^{i} \times N_{\mathrm{bkg}}$, and the observed number of events $\left(n_{i}\right)$ in the dijet mass distribution. A multiplication of the Poisson probabilities for all mass bins completes the $L H$, and the maximum $L H$ returns the most probable number of $H^{+}, W$ and non- $t \bar{t}$ background in the given dijet mass distribution: $N_{W}, N_{H^{+}}$, and $N_{\text {bkg }}$. In the $L H$ fit, $N_{W}$ and $N_{H^{+}}$float without limits, but the non$t \bar{t}$ backgrounds $\left(N_{\mathrm{bkg}}\right)$ are constrained within Gaussian uncertainty $\left(\sigma_{\mathrm{N}_{\mathrm{bkg}}}\right)$. Based on the $L H$ fit output, the $\mathscr{B}\left(t \rightarrow H^{+} b\right)$ is calculated assuming $\mathscr{B}\left(t \rightarrow H^{+} b\right)+\mathscr{B}(t \rightarrow W b)=1$ and $\mathscr{B}\left(H^{+} \rightarrow c \bar{s}\right)$ $=1$.

\section{Incorporating Systematic Uncertainties \& Setting Upper Limits}

There are a number of systematic error sources that affect the resulting $\mathscr{B}\left(t \rightarrow H^{+} b\right)$ : jet energy scale corrections [12], initial state and final state radiation, $W+$ jets production scale, the choice of simulation program (PYTHIA vs. HERWIG [13]), and $b$-tagging scale factor (the ratio of $b$-tagging efficiency $\left.\varepsilon_{\mathrm{DATA}} / \varepsilon_{\mathrm{MC}}\right)$. These systematic sources, except for the $b$-tagging scale factor, perturb the dijet mass shape, and cause a shift in the $L H$ fit results, $\mathscr{B}\left(t \rightarrow H^{+} b\right)$. The $\mathscr{B}(t \rightarrow$ $H^{+} b$ ) shift is estimated from the $L H$ fits to perturbed and un-perturbed pseudoexperiments; the pseudoexperiments are generated from a bin-by-bin Poisson fluctuation to the perturbed and unperturbed dijet mass distributions of the SM processes. The $\mathscr{B}\left(t \rightarrow H^{+} b\right)$ shift from each source provides an estimate of the systematic uncertainty. On the other hand, $\pm 1 \sigma$ shift on the $b$-tagging scale factor causes the selection acceptances of the $H^{+}$and $W$ events to change, thus affecting the 


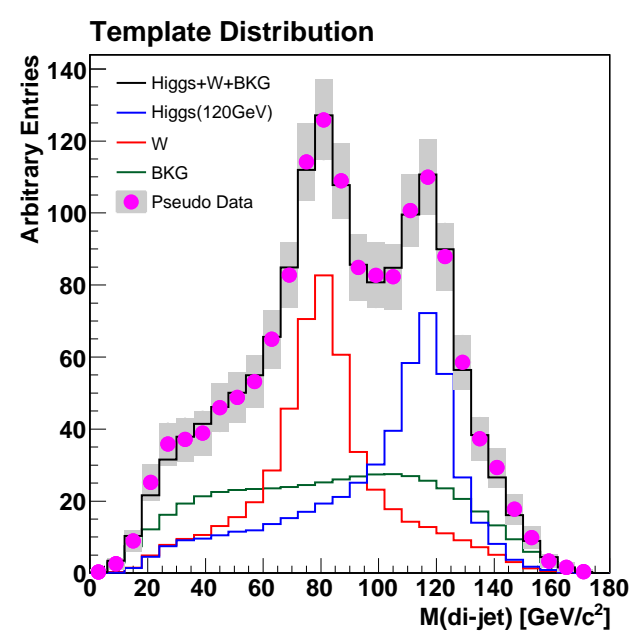

Figure 3: A template consists of the dijet mass of $H^{+}\left(120 \mathrm{GeV} / \mathrm{c}^{2}\right), W$, and non- $t \bar{t}$ background. A dummy pseudoexperiment containing $N_{W}=N_{H^{+}}=N_{n o n-t \bar{t}}$ is fit with the template, and shows its components.

$\mathscr{B}\left(t \rightarrow H^{+} b\right)$ calculation. The individual systematic uncertainties are combined in quadrature, and the total systematic uncertainty is shown as a function of $\mathscr{B}\left(t \rightarrow H^{+} b\right)$ in Fig. 4.

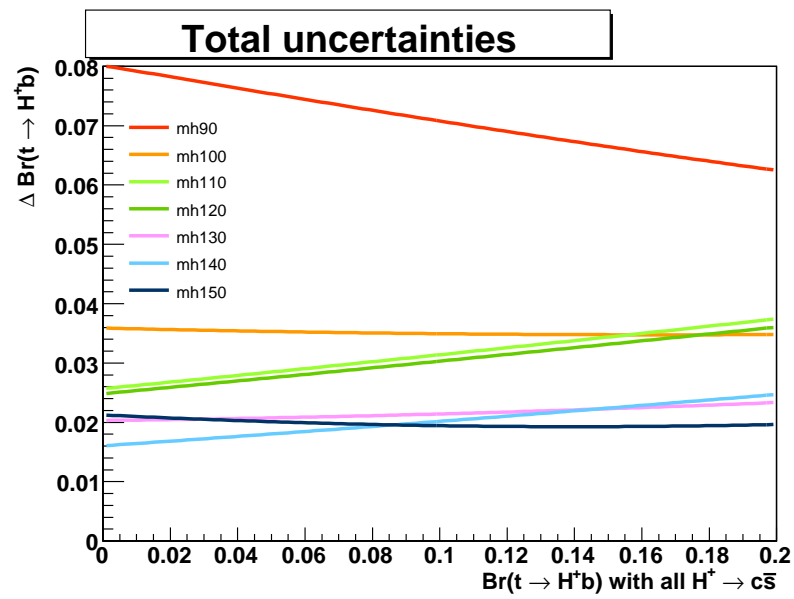

Figure 4: Total systematic uncertainty $\left(\Delta \mathscr{B}\left(t \rightarrow H^{+} b\right)\right)$ shown as a function of $\mathscr{B}\left(t \rightarrow H^{+} b\right)$ for $H^{+}$masses of 90 to $150 \mathrm{GeV} / c^{2}$.

The total systematic uncertainty $\Delta \mathscr{B}\left(t \rightarrow H^{+} b\right)$ is used for an input error in the Gaussian smearing of the $L H$ as a function of $\mathscr{B}\left(t \rightarrow H^{+} b\right)$ :

$$
L H^{\prime}\left(x^{\prime}\right)=\int_{0}^{1} L H(x) \times \frac{1}{\Delta\left(x^{\prime}\right) \sqrt{2 \pi}} \exp \left(-\frac{1}{2}\left(\frac{x^{\prime}-x}{\Delta\left(x^{\prime}\right)}\right)^{2}\right) d x .
$$

Here the $L H(x)$ is a direct likelihood fit result as a function of $x$. Both $x$ and $x^{\prime}$ stand for the branching ratio, $\mathscr{B}\left(t \rightarrow H^{+} b\right)$, and vary between 0 and $1 . \Delta\left(\mathrm{x}^{\prime}\right)$ is the total systematic uncertainty 
at $\mathrm{x}^{\prime}$. The smeared likelihood $L H^{\prime}\left(x^{\prime}\right)$ is computed from $L H(x)$ in convolution with the Gaussian probability for the entire $x$ range. The upper limit on $\mathscr{B}\left(t \rightarrow H^{+} b\right)$ with $95 \%$ confidence level (C.L.) is estimated by one-sided integration of $L H^{\prime}\left(x^{\prime}\right)$ up to $95 \%$ of the positive $\mathscr{B}\left(t \rightarrow H^{+} b\right)$ area. Repeating this process on a thousand null-Higgs pseudoexperiments provides the expected upper limit in the SM with 1, $2 \sigma$ statistical fluctuations.

\section{Results}

The observed dijet mass distribution in the $2.2 \mathrm{fb}^{-1}$ CDF II data is compared with the SM expectations in Fig. 5. There is no significant excess observed, hence the 95\% C.L. upper limits are placed at $\mathscr{B}\left(t \rightarrow H^{+} b\right)<0.1$ to 0.3 for the $H^{+}$masses of 90 to $150 \mathrm{GeV} / c^{2}$ in Fig. 6 . This result shows that the observed upper limits from data agree with the SM expectation.

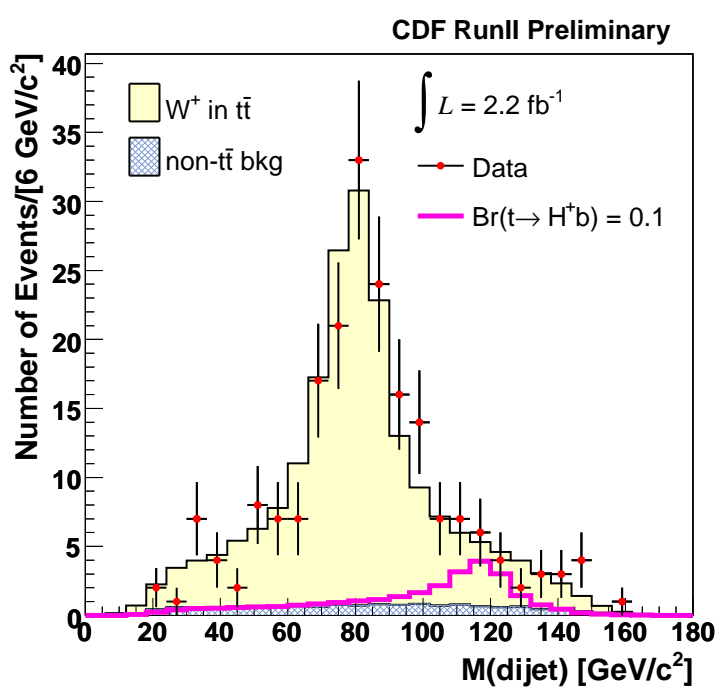

Figure 5: Observed dijet mass in top quark decays is compared with the SM expectation, a stack of $W$ bosons and non- $t \bar{t}$ backgrounds in the $2.2 \mathrm{fb}^{-1} \mathrm{CDF}$ II data sample. An example distribution of the 120 $\mathrm{GeV} / \mathrm{c}^{2} H^{+}$boson is overlaid assuming $\mathscr{B}\left(t \rightarrow H^{+} b\right)=0.1$, which corresponds to the $95 \%$ C.L. upper limit on $\mathscr{B}\left(t \rightarrow H^{+} b\right)$.

\section{Acknowledgment}

I thank the organizers at Uppsala University for the invitation and productive workshops. I also thank to the CDF colleagues and Fermilab staffs for their efforts to bring this results out.

\section{References}

[1] P. W. Higgs, Phys. Lett. 12, 132 (1964); Phys. Rev. Lett. 13, 508 (1964); Phys. Rev. 145, 1156 (1966); F. Englert and R. Brout, Phys. Rev. 145, 1156 (1964); G. S. Guralnik, C. R. Hagen, and T. W. B. Kibble, Phys. Rev. Lett. 13, 585 (1964). 


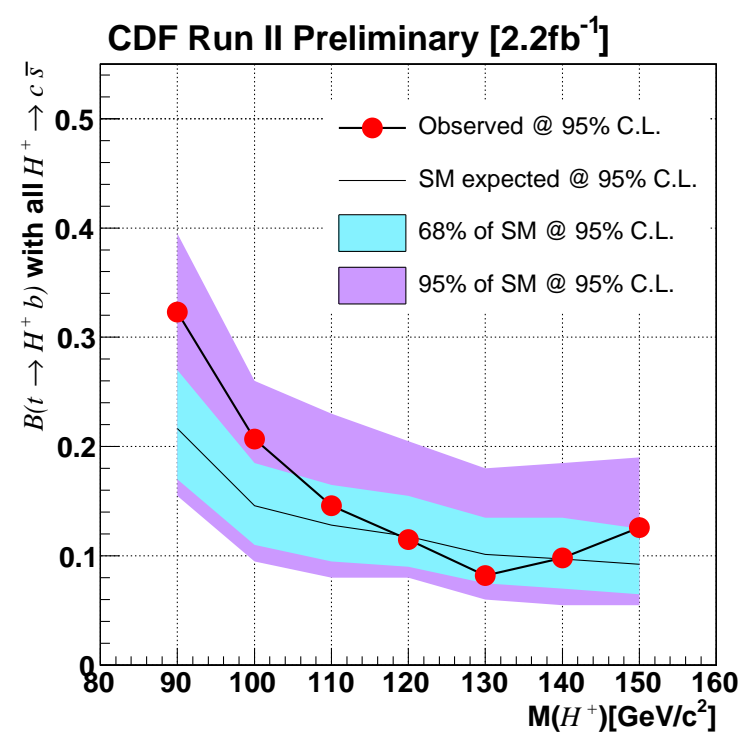

Figure 6: The upper limits on the $\mathscr{B}\left(t \rightarrow H^{+} b\right)$ at $95 \%$ C.L. The observed upper limits from the data are compared to the SM expectations for the $H^{+}$masses of 90 to $150 \mathrm{GeV} / \mathrm{c}^{2}$.

[2] H. E. Haber, G. Kane, and S. Dawson, The Higgs Hunter's Guide (Addison-Wesley, Reading, MA, 1990).

[3] F. Abe et al. (CDF Collaboration), Phys. Rev. Lett. 74, 2626 (1995); S. Abachi et al. (DØ Collaboration), Phys. Rev. Lett. 74, 2632 (1995).

[4] A. Abulencia et al., The CDF Collaboration, Phy. Rev. Lett. 96 042003(2006)

[5] M. Carena, S. Heinemeyer, C.E.M. Wagner, and G. Weiglein, hep-ph/9912223.

[6] The CDF II Detector Technical Design Report, Fermilab-Pub-96/390-E.

[7] D. Acosta et al. (CDF Collaboration), Phys. Rev. D 71, 052003 (2005).

[8] MINUIT - Function Minimization and Error Analysis, CERN Program Library entry D506.

[9] M. Cacciari, S. Frixione, G. Ridolfi, M. Mangano and P. Nason, J. High Energy Phys. 404, 68 (2004); N. Kidonakis and R. Vogt, Phys. Rev. D 68, 114014 (2003).

[10] T. Sjostrand et al., Comput. Phys. Commun. 135, 238 (2001).

[11] Michelangelo L. Mangano et al., J. High Energy 307 Phys. 001 (2003).

[12] A. Bhatti et al., Nucl. Instrum. Methods. A 566, 375 (2006).

[13] G. Corcella et al., J. High Energy Phys. 10110 (2001). 